\title{
Testing of kisspeptin levels in girls with idiopathic central precocious puberty and its significance
}

\author{
YU YANG, XIANG-YU XIONG, LI YANG, LILING XIE and HUI HUANG \\ Department of Endocrinology, The Children's Hospital of Jiangxi, Nanchang, Jiangxi 330006, P.R. China
}

Received June 25, 2014; Accepted March 3, 2015

DOI: $10.3892 /$ etm.2015.2398

\begin{abstract}
The aim of this study was to explore the significance of plasma kisspeptin levels in diagnosis and therapeutic evaluation through the analysis of the kisspeptin levels of girls diagnosed with idiopathic central precocious puberty (ICPP) prior to treatment and after 6-months of treatment and those with simple premature thelarche (PT). A total of 70 girls including 24 girls diagnosed with ICPP, 21 girls with PT and 25 normal girls were enrolled in the study. ELISA analysis was conducted to detect the plasma levels of kisspeptin. The kisspeptin level of the ICPP group prior to treatment $(1.80 \pm 0.13 \mathrm{ng} / \mathrm{ml})$ was significantly higher than those of the other two groups. The kisspeptin level of the ICPP group after 6 months of treatment $(1.49 \pm 0.21 \mathrm{ng} / \mathrm{ml})$ was significantly lower than that prior to treatment $(\mathrm{P}<0.05)$. It may be concluded that the plasma level of kisspeptin is associated with the initiation of pubertal development, and it may serve as an important parameter in the diagnosis of ICPP and the evaluation of therapeutic effects.
\end{abstract}

\section{Introduction}

It is generally accepted that precocious puberty (PP) refers to signs of sexual development occurring in girls younger than 8 years of age or boys younger than 9 years of age, or the appearance of menstruation in girls before the age of 10 years. The major manifestations of PP include a growth spurt, together with maturity of the reproduction organs and sexual characteristics that are significantly advanced compared with those of other children of the same age. In girls, idiopathic central precocious puberty (ICPP) accounts for $>80 \%$ of the cases of central precocious puberty (CPP).

The clinical diagnosis of this condition and the evaluation of therapeutic effects are mainly conducted through physical

Correspondence to: Dr Yu Yang, Department of Endocrinology, The Children's Hospital of Jiangxi, 122 Yangming Road, Nanchang, Jiangxi 330006, P.R. China

E-mail: docyuyang@163.com

Key words: kisspeptin, idiopathic central precocious puberty, simple premature thelarche examination, and the assessment of bone age and growth rate. Currently, owing to the high accuracy of provocation testing with hypothalamic gonadotropin releasing hormone (GnRH), it continues to be the gold standard in the diagnosis of CPP with active suppression of gonadal axis post-treatment. However, provocation testing with GnRH is not yet widely used due to it being expensive, time-consuming and causing an uncomfortable sensation similar to that of repeated hemospasia/pricking.

The pathogenesis of ICPP is currently unclear. Previous studies have confirmed that the kisspeptin/G protein-coupled receptor 54 (GPR54) signaling pathway initiates the onset of puberty $(1,2)$, which is probably closely associated with the occurrence and development of ICPP. The early diagnosis of $\mathrm{PP}$ and evaluation of therapeutic effects is very important; however, to the best of our knowledge, no studies concerning the kisspeptin levels of normal children, girls with premature thelarche (PT) and girls with ICPP ante-and-post treatment have been reported.

The purpose of present study was to explore the role of kisspeptin in the initiation of pubertal growth and its significance in the diagnosis of ICPP in girls and the evaluation of the effects of ICPP therapy. The kisspeptin levels of girls with ICPP ante- and post-treatment, girls with PT, and normal girls of the same age were compared with the aim of elucidating the correlation of the kisspeptin signaling mechanism with suppression of the gonadal axis in girls with ICPP at onset and post-treatment. This may facilitate the development of new guidelines for the early diagnosis of ICPP in females and the evaluation of therapeutic effects.

\section{Materials and methods}

\section{Subjects}

A case-control study design was adopted. Informed consent was obtained from the subjects and their guardians and approval was provided by ethics committee of the Children's Hospital of Jiangxi (Nanchang, China).

ICPP group. A total of 24 girls diagnosed with ICPP in this hospital from June 2012 to January 2013 formed the ICPP group. The diagnosis standard of ICPP was in accordance with the diagnosis and treatment guidelines of central (true) precocious puberty as established by the Subspecialty Group of Endocrinology, Hereditary and Metabolic Diseases of the Society of Pediatrics, Chinese Medical Association in 2007 (3). 
Normal control group. The normal control group comprised girls who accepted a physical health examination at the above period in this hospital. The inclusion criteria were: i) no manifestation of the development of sexual characteristic; ii) height and weight between the mean \pm 2 standard deviations (SD) of those of children with the same gender and of the same age; iii) age matched as closely as possible with the ICPP group ( \pm 1 year).

PT group. The PT group comprised girls diagnosed with PT in this hospital during the aforementioned time period. The inclusion criteria were: i) only premature thelarche, without occurrence of other secondary sex characteristics, and no coloring of the areola of the breast; ii) followed up for 1 year, showing a non-progressive self-limited course; iii) height and weight between mean $\pm 2 \mathrm{SD}$ of those of children with the same gender and of the same age; iv) age matched as closely as possible with the ICPP group ( \pm 1 year).

\section{Research methods}

Growth and development evaluation. Height, weight, body mass index (BMI) and Tanner staging for girls were determined by clinical specialists to evaluate the puberty developmental level. Bone age determination via the Tanner-Whitehouse (TW2) method (4) and B ultrasonic examination of the ovaries and uterus were conducted.

Detection of baseline levels of luteinizing hormone ( $\mathrm{LH})$, follicle-stimulating hormone (FSH) and estradiol $\left(E_{2}\right)$. A chemiluminescence method was adopted to measure the levels of LH, FSH and $\mathrm{E}_{2}$ using kits obtained from Siemens Healthcare Diagnostics Inc., (Tarrytown, NY, USA).

Provocation testing with GnRH. Gonadorelin was administered intravenously according to a dosage level of $2.5 \mu \mathrm{g} / \mathrm{kg}$ (maximum dose, $100 \mathrm{~g}$ ). Serum LH and FSH levels were detected at $0,30,60$ and 90 min after the injection using a chemiluminescence-based method, as described above. In this test, a peak stimulated $\mathrm{LH}$ level of $>3.3-5.0 \mathrm{U} / 1$ is considered to indicate a diagnosis of CPP, and a ratio of $\mathrm{LH} / \mathrm{FSH}>0.6$ may be diagnosed as CPP.

Exclusion of ICPP due to organic causes. For girls considered to have ICPP, blood, urine and feces routine tests were conducted. Tests of hepatic, renal and thyroid gland function were also conducted, and the levels of adrenocorticotropic hormone, growth hormone and $17 \alpha$-hydroxyprogesterone were detected. Abdominal B ultrasound scanning of the liver, gallbladder, spleen, pancreas, kidneys and adrenal gland together with magnetic resonance imaging of the hypothalamus and hypophysis were conducted to exclude ICPP due to organic causes.

Detection of the plasma kisspeptin level by ELISA. A 1-ml specimen of blood was put into an EDTA anticoagulant tube containing 0.6TI aprotinin (Amresco; Shanghai Haoran Bio Technologies Co., Ltd., Shanghai, China), immediately mixed gently, allowing aprotinin to contact the blood sufficiently to reduce the degradation of kisspeptin by protease, and then conserved at $4^{\circ} \mathrm{C}$. The blood specimen was centrifuged for $15 \mathrm{~min}$ at $1,600 \times \mathrm{g}, 4^{\circ} \mathrm{C}$ and the supernatant was extracted and conserved at $-80^{\circ} \mathrm{C}$ for detection. All of the above procedures were completed within $1 \mathrm{~h}$. The plasma specimen was then loaded onto a C18-Sep-Column (Bejing H\&E Technology Co., Ltd., Beijing, China), and the eluent was evaporated and dried using a SIM FD-5 vacuum freeze dryer (SIM International Group Co., Ltd., Shanghai, China), then reconstituted. The kisspeptin level was detected by ELISA according to directions of the kisspeptin test kit (Phoenix Pharmaceuticals, Inc., Burlingame, CA, USA).

ICPP treatment and evaluation of therapeutic effect. i) Treatment. The first dosage of triptorelin was administered according to a dosage level of $80-100 \mu \mathrm{g} / \mathrm{kg}$ (maximum dose, $3.75 \mathrm{mg}$ ). Subsequently, one injection was given every 4 weeks. For those patients whose weight was $>30 \mathrm{~kg}, 3.75 \mathrm{mg}$ triptorelin was injected intramuscularly every 4 weeks, and the maintenance dosage was adjusted according to the suppression of gonadal axis function (including sexual characteristics, sex hormone levels and bone age).

ii) Therapeutic effect evaluation. Height and the status of sexual characteristics development were determined every 3-6 months during the course of treatment. The GnRH provocation test was readministered at 3-6 months after the first dose. Serum $\mathrm{E}_{2}$ level measurements, B ultrasound scanning of the ovaries and uterus, and bone age evaluation were conducted after 3 months of treatment. Plasma kisspeptin levels were monitored after 6 months of treatment (3).

Statistical analysis. Statistical analyses were performed using SPSS for Windows, version 18.0 (SPSS, Inc., Chicago, IL, USA). All test results are presented as the mean \pm standard deviation. Student's $t$-test was used for pairwise comparison of measurement data. Differences of multiple sample rates were compared by analysis of variance. The correlation of kisspeptin levels in different groups was analyzed by Pearson correlation analysis. Height is expressed as SDS, which was calculated as follows: $\mathrm{SDS}=$ (measured value - mean $) / \mathrm{SD}$. $\mathrm{P}<0.05$ was considered to indicate a statistically significant difference.

\section{Results}

Comparison of clinical parameters and growth among the different groups. A total of 24 girls with ICPP treatment, 21 girls with PT and 25 normal girls as the control were enrolled in this study. The average age at the first diagnosis was $7.53 \pm 0.71$ years in the ICPP group, $7.62 \pm 1.42$ years in the PT group and 7.27 \pm 1.51 years in the control group. The healthy control group comprised undeveloped prepubertal girls, and they had the lowest BMI $\left(15.02 \pm 1.93 \mathrm{~kg} / \mathrm{m}^{2}\right)$. The BMI for girls with ICPP prior to treatment was the highest $\left(17.09 \pm 3.05 \mathrm{~kg} / \mathrm{m}^{2}\right)$, which indicated that the BMI increased along with pubertal development. Compared with the PT and control groups, the girls in the ICPP group had significantly advanced bone age. The ratio of bone age/actual age was $1.57 \pm 0.64(\mathrm{~F}=114.1, \mathrm{P}<0.001)$. The SDS of height suggested clearly accelerated growth in the ICPP group (1.05 \pm 0.78 ; $\mathrm{F}=135, \mathrm{P}<0.001)$. The $\mathrm{E}_{2}$ level and volume of the ovaries and uterus in girls of the ICPP group were also markedly higher than those of other two groups, with the difference being statistically significant $(\mathrm{P}<0.001$; Table I).

Comparison of plasma kisspeptin levels among different groups. The average plasma kisspeptin level of the ICPP group at the time of diagnosis $(1.80 \pm 0.13 \mathrm{ng} / \mathrm{ml})$ was higher 


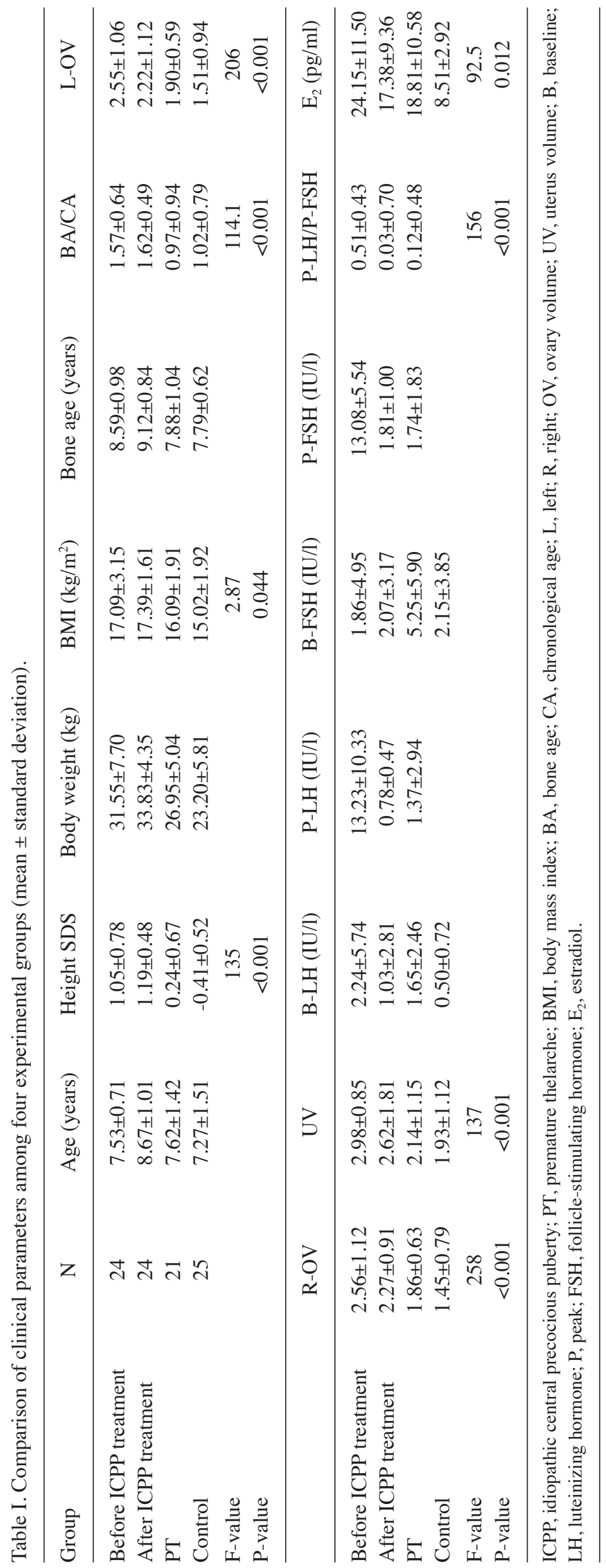




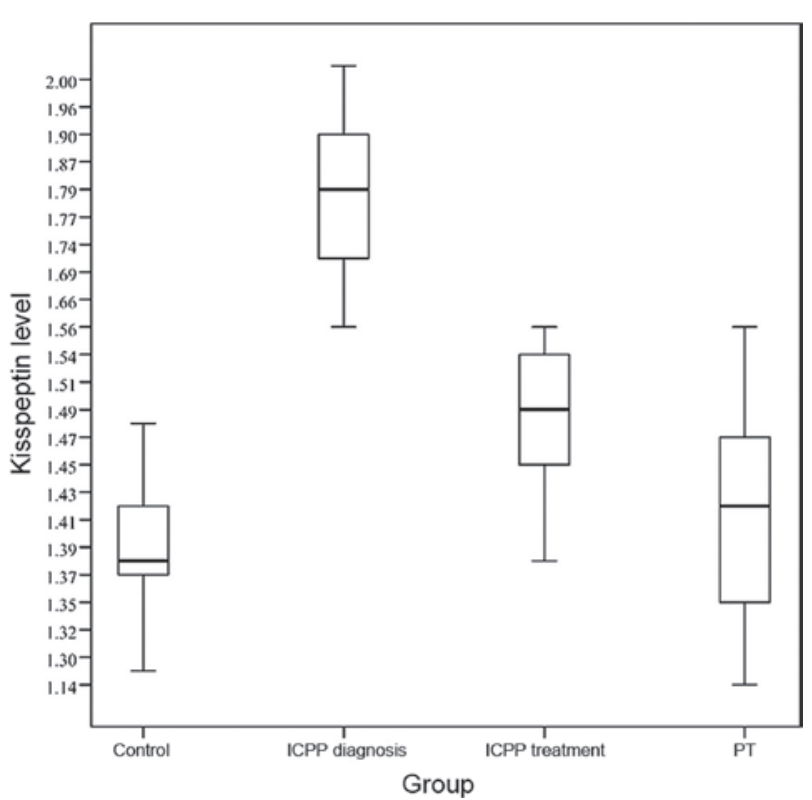

Figure 1. Kisspeptin level of girls with ICPP at the time of diagnosis and after 6 months of treatment, and those of girls in PT group and control group. CPP, idiopathic central precocious puberty; PT, premature thelarche.

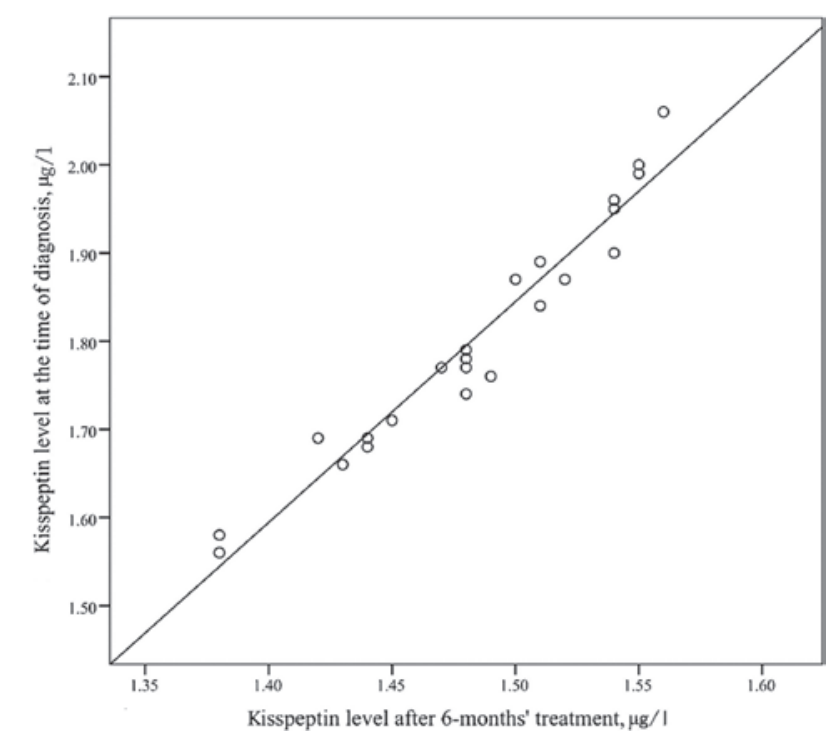

Figure 2. Positive correlation of kisspeptin levels at the time of ICPP diagnosis and at follow-up. ICPP, idiopathic central precocious puberty.

than those of the PT group $(1.41 \pm 0.10 \mathrm{ng} / \mathrm{ml})$ and the control group $(1.39 \pm 0.13 \mathrm{ng} / \mathrm{ml})$, with the differences being statistically significant $(\mathrm{F}=109.7, \mathrm{P}<0.001$; Fig. 1). When pubertal development in the ICPP group was completely inhibited after 6 months of treatment, plasma kisspeptin levels $(1.49 \pm 0.15 \mathrm{ng} / \mathrm{ml})$ were clearly decreased compared with those prior to treatment $(1.80 \pm 0.13 \mathrm{ng} / \mathrm{ml})$, and the difference was statistically significant $(\mathrm{t}=10.80, \mathrm{P}<0.001$; Fig. 1$)$. The difference in kisspeptin levels between the PT group and the control group was not statistically significant $(\mathrm{t}=10.97$, $\mathrm{P}=0.095)$. The kisspeptin level at the time of ICPP diagnosis and the kisspeptin level after treatment were positively correlated in a statistically significant manner $(\mathrm{r}=0.974, \mathrm{P}<0.001$; Fig. 2).

\section{Discussion}

The pubertal development of humans is a complex process associated with hormone secretion, which is regulated by the hypothalamic-pituitary-gonadal (HPG) axis. The activation of the HPG axis is under integrated regulation by genetics, body energy status and environmental and other factors (5). Activation of GnRH neurons is the key to triggering the initiation of puberty. A previous study has shown that at peri-puberty, very high Kiss-1 and GPR54 mRNA levels are detectable in the rat hypothalamus, and Kiss-1 gene expression levels are increased in the anteroventral periventricular nuclei (6), which suggests that kisspeptin may play an important role in the regulation of GnRH secretions and may act directly on GnRH neurons (7).

Pielecka-Fortuna and Moenter (8) observed that the intraventricular injection of kisspeptins into mice promoted the release of $\mathrm{GnRH}$, and a parallel increase in plasma LH occurred. Plant, Ramaswamy and Dipietro (9) repeatedly administered kisspeptin-10 to juvenile monkeys intravenously and GnRH secretion, indicating sexual maturity, occurred prematurely. These findings imply that the secretion of kisspeptin has a close association with the initiation of puberty.

These data indicate the important role of kisspeptin in pubertal development. However, the majority of studies have focused on the role of kisspeptin in the activation of the HPG axis and its mechanism. Studies concerning the clinical application of kisspeptin are rare.

In the present study, the kisspeptin levels of girls with ICPP were detected prior to treatment and following 6 months of treatment, and were compared with those of girls with PT and healthy controls. The results demonstrated that the kisspeptin levels of girls in the ICPP group prior to treatment were significantly higher than those of the other groups, which supports the hypothesis that kisspeptin is an activating effector of the HPG axis. Significant increases in Kiss-1 gene expression trigger the pulsed release of GnRH, activate the HPG axis and initiate pubertal development, thereby causing the occurrence of PP (10).

Studies on the blood levels of kisspeptin in children have particularly focused on comparisons between girls with CPP and those with PT or normal undeveloped girls, and there have been no comparative studies on the plasma kisspeptin levels of girls with ICPP prior to and following treatment in China. In the present study, the kisspeptin levels of girls with ICPP before treatment and after 6 months of treatment were compared, and the results revealed that the kisspeptin level of the ICPP group decreased significantly during this treatment period. Based on this result, combined with a significant reduction of the $\mathrm{LH}$ peak/FSH peak ratio in the GnRH provocation test when checked after treatment, it is considered that the HPG axis was suppressed by the treatment and the expression of Kiss-1 gene fell to a level typical of ante-pubertal development. As for comparison of the ICPP group after treatment with the control group, the results of the two groups were similar, and the kisspeptin level following ICPP treatment was only slightly higher than that of the control. However, the average age of the ICPP group post-treatment was nearly 1.5 years greater than that of the control group. Further research is required to assess 
whether kisspeptin levels could decrease to the same level as those of the control group or lower with continued treatment.

Demirbilek et al (11) detected the plasma kisspeptin levels of 28 girls with ICPP prior to treatment and after 6 months of treatment, and the results demonstrated that the kisspeptin level was clearly decreased following the treatment, which is consistent with the results of the present study. In addition, statistical analysis showed that the plasma kisspeptin levels of girls with ICPP prior to and following treatment were positively correlated, all of which implies that the plasma level of kisspeptin could be used an indicator to evaluate the therapeutic effects in the treatment of precocious puberty.

Liu et al (12) detected the plasma kisspeptin levels of 20 healthy girls at the Tanner I stage of development and 20 girls with ICPP at the Tanner II and III stages; the results showed that the kisspeptin levels of girls with ICPP were clearly higher than those of the healthy controls, and were positively correlated with LH peak value and bone age. A study of kisspeptin levels in 30 girls with CPP and 30 undeveloped girls of the same age conducted by Rhie et al (13) confirmed this outcome. Ma et al (14) detected the plasma kisspeptin levels of girls with ICPP, girls with PT and normal girls at Tanner stages I-V. The results showed that the plasma kisspeptin levels of girls with ICPP were significantly increased compared with those of girls with PT. Kisspeptin levels at the Tanner II stage of pubertal development were significantly higher than those at other Tanner stages; moreover, the kisspeptin level began to gradually decrease following the Tanner II stage, which demonstrated that the Kiss-1/GPR54 system has a switching effect on pubertal development. Activation of the Kiss-1 gene is probably the key to triggering the initiation of puberty, and Kiss-1 gene expression in the HPG axis may be affected by regulatory feedback from sex hormones. Kisspeptin levels gradually decline as pubertal development progresses (15). In the present study, the kisspeptin level of girls with ICPP prior to treatment was significantly higher than that of the PT group, which implies that pubertal development in the ICPP group has been initiated and the Kiss-1 gene activated, and demonstrates that the measurement of kisspeptin levels has some significance in the early identification of ICPP and PT.

A study of 20 girls with normal development and 20 girls with PT by Akinci, Cetin and Ilhan (16) demonstrated that the plasma kisspeptin level in the girls with PT was significantly higher than that of the girls with normal development and was correlated with the prolactin level. In the present study, the plasma kisspeptin level of the PT group was similar to that of the control group; no significantly statistical difference was identified. The age difference between the two groups was 1.5 years, and the kisspeptin levels of the two groups were not measured following PT treatment. Therefore, it is necessary to expand the sample size in order to investigate whether kisspeptin can be used in the auxiliary diagnosis of PT.

In conclusion, plasma kisspeptin levels rise at the initiation of pubertal development, and the kisspeptin levels of girls with PP decline following treatment with $\mathrm{GnRH}$, which indicates that the detection of kisspeptin can contribute to the early diagnosis of ICPP and may be used as an indicator of therapeutic effects in the treatment of precocious puberty. The results of the study support the viewpoint that the
Kiss-1/GPR54 gene is the key to regulating the initiation of pubertal development and that kisspeptin is closely associated with the initiation and development of ICPP (17). Research into kisspeptin is continuing, and the clinical use of kisspeptin for ICPP diagnosis and the evaluation of the effectiveness of therapy remains to be further explored.

\section{Acknowledgements}

The abstract was presented as a poster (P1-D3-224) at the 53rd Annual Meeting of the European Society for Paediatric Endocrinology (ESPE), Sep 18-20, 2014 in Dublin, Ireland.

\section{References}

1. Roa J, Navarro VM and Tena-Sempere M: Kisspeptins in reproductive biology: Consensus knowledge and recent developments. Biol Reprod 85: 650-660, 2011.

2. Sonigo C and Binart N: Overview of the impact of kisspeptin on reproductive function. Ann Endocrinol (Paris) 73: 448-458, 2012.

3. Subspecialty Group of Endocrinology: Hereditary and Metabolic Diseases, Society of Pediatrics, Chinese Medical Association: The diagnosis and treatment guidelines of central (true) precocious puberty. Zhonghua Er Ke Za Zhi 45: 426-427, 2007 (In Chinese).

4. Murray RO: Assessment of Skeletal Maturity and Prediction of Adult Height (TW2 Method). Proc R Soc Med 69: 542, 1976.

5. Yang L, Tang K, Qi Y, et al: Potential metabolic mechanism of girls' central precocious puberty: A network analysis on urine metabonomics data. BMC Syst Biol 6 (Suppl 3): S19, 2012.

6. Takumi K, Iijima N and Ozawa $\mathrm{H}$ : Developmental changes in the expression of kisspeptin mRNA in rat hypothalamus. J Mol Neurosci 43: 138-145, 2011.

7. Gopurappilly R, Ogawa S and Parhar IS: Functional significance of $\mathrm{GnRH}$ and kisspeptin and their cognate receptors in teleost reproduction. Front Endocrinol (Lausanne) 4: 24, 2013.

8. Pielecka-Fortuna $\mathbf{J}$ and Moenter SM: Kisspeptin increases gamma-aminobutyric acidergic and glutamatergic transmission directly to gonadotropin-releasing hormone neurons in an estradiol-dependent manner. Endocrinology 151: 291-300, 2010.

9. Plant TM, Ramaswamy S and Dipietro MJ: Repetitive activation of hypothalamic $\mathrm{G}$ protein-coupled receptor 54 with intravenous pulses of kisspeptin in the juvenile monkey (Macaca mulatta) elicits a sustained train of gonadotropin-releasing hormone discharges. Endocrinology 147: 1007-1013, 2006.

10. Dedes I: Kisspeptins and the control of gonadotrophin secretion. Syst Biol Reprod Med 58: 121-128, 2012.

11. Demirbilek H, Gonc EN, Ozon A, Alikasifoglu A and Kandemir N: Evaluation of serum kisspeptin levels in girls in the diagnosis of central precocious puberty and in the assessment of pubertal suppression. J Pediatr Endocrinol Metab 25: 313-316, 2012.

12. Liu F, Zeng YE, Xia HB, et al: Change of serum kisspeptins and its significance in girls with idiopathic central precocious puberty. Shi Yong Er Ke Lin Chuang Za Zhi 26: 1558-1559, 2011 (In Chinese).

13. Rhie YJ, Lee KH, Eun SH, et al: Serum kisspeptin levels in Korean girls with central precocious puberty. J Korean Med Sci 26: 927-931, 2011.

14. Ma XY, Ni JH, Liu MZ, et al: Plasma kisspeptin levels in normal female pubertal stages and in girls with idiopathic central precocious puberty. Zhonghua Nei Fen Mi Dai Xie Za Zhi 27: 36-39, 2011 (In Chinese).

15. Yang $Y$ and Xiong XY: Study progress of central precocious puberty and KISS-1/G protein-coupled receptor 54 gene. Shi Yong Er Ke Lin Chuang Za Zhi 27: 1548-1551, 2012 (In Chinese).

16. Akinci A, Cetin D and Ilhan N: Plasma kisspeptin levels in girls with premature thelarche. J Clin Res Pediatr Endocrinol 4: 61-65, 2012 .

17. Rønnekleiv OK and Kelly MJ: Kisspeptin excitation of GnRH neurons. Adv Exp Med Biol 784: 113-131, 2013. 